\title{
Medial Preoptic Sexual Dimorphisms in the Guinea Pig. II. An Investigation of Medial Preoptic Neurogenesis
}

\author{
William Byne, John T. Warren, and Inge Siggelkow \\ Neurosciences Training Program and Department of Neurophysiology, University of Wisconsin, Madison, Wisconsin 53706
}

\begin{abstract}
Neurogenesis was studied in the medial preoptic area of the guinea pig by the method of tritiated thymidine autoradiography. Eight cytoarchitectonic divisions were examined, 4 of which display sexual dimorphism and 4 that do not. Neurogenesis in the nonsexually dimorphic divisions was found to end at embryonic day (E) 27 , while in each of the sexually dimorphic divisions neurogenesis continued after this and persisted until at least E31 in the central compact and principal portions of the medial preoptic nucleus. Since the testes become active at E25 in the guinea pig (Resko, 1970), it is possible that gonadal secretions influence the proliferation and subsequent developmental processes of neurons destined for the sexually dimorphic nuclei.
\end{abstract}

The medial preoptic area (MPA) of the guinea pig exhibits prominent sexually differentiated patterns of cell density and distribution (Bleier et al., 1982). The most conspicuous sexual dimorphisms involve the medial preoptic nucleus (MPN) as described by Byne and Bleier (1987). This nucleus has 3 divisions: (1) an anterior compact subnucleus (MPNa) that occupies a larger volume in females than males; (2) a central compact subnucleus (MPNc) that occupies a larger volume in males and corresponds cytoarchitectonically to the sexually dimorphic nucleus of the preoptic area (SDN-POA) described by Gorski et al. (1978) in the rat; and (3) the principal portion of the MPN (MPNp), which is moderately cellular and in which the densely cellular MPNa and MPNc are embedded. In addition to the sex differences in the MPN, at some levels the anterior periventricular nucleus (Pea) appears more densely cellular in females, while portions of the bed nucleus of the stria terminalis (BNST) arc more prominent in males (Bleier et al., 1982; Hines et al., 1985). The sex differences in MPNa and MPNc have been shown to develop largely in response to sex differences in prenatal androgen exposure, while the prenatal hormonal dependence of the other dimorphisms has not been investigated (Byne and Bleier, 1987).

As a first step toward elucidating the mechanisms by which androgens and their metabolites influence hypothalamic cy-

\footnotetext{
Received Sept. 23, 1986; revised Feb. 23, 1987; accepted Feb. 26, 1987.

W.B. was a predoctoral fellow in the Neurosciences Training Program at the University of Wisconsin at the time of this study. We are very grateful to Joan Meister and Jo Ann Ekleberry for their careful and skillful histological work, and to Ruth Bleier, Robert Goy, Ei Terasawa, and Melissa Hines for their suggestions and careful reading of the original manuscript. This work has been supported by grants from the NIH (NS16643 and HD03352), the University of Wisconsin Graduate School (to R.B.) and by neurosciences training grant NIH GM-07507 (to W.B.).

Correspondence should be addressed to William Byne, Albert Einstein College of Medicine, Box 52, 1300 Morris Park Avenue, Bronx, NY 10461.

Copyright (C) 1987 Society for Neuroscience $0270-6474 / 87 / 092697-06 \$ 02.00 / 0$
}

toarchitecture, the present study was undertaken to determine the period during which MPA neurons are formed using the method of tritiated thymidine ( $\left.{ }^{3} \mathrm{HT}\right)$ autoradiography. The theoretical aspects of this technique have been discussed extensively by Sidman (1970). Briefly, since thymidine is a precursor of DN $\Lambda$, the tritiated compound is incorporated into the nuclei of dividing cells that are exposed to it, and since DNA replication is semiconservative, half of the label originally incorporated into the nucleus of a cell is lost from that cell with each subsequent division. ${ }^{3} \mathrm{HT}$, therefore, permanently and substantially labels only those cells undergoing one of their final divisions at the time of exposure.

Neurogenesis within the hypothalamus has been studied extensively in the rat (Ifft, 1972; Altman and Bayer, 1978a, b; Jacobson and Gorski, 1981), but to date no such studies in the guinea pig have been reported. The present study describes the patterns of neurogenesis of the sexually dimorphic nuclei and several adjacent nuclei in the MPA of the guinea pig. Neuronal proliferation was found to continue in the sexually dimorphic cell groups after it had ceased in their neighboring nuclei.

\section{Materials and Methods}

Mature female albino guinea pigs of Hartley stock were time-mated as described elsewhere (Byne and Bleier, 1987) and the day of mating designated as day 0 . On a single day of gestation between embryonic days (E) 20 and 31, at $0900 \mathrm{hr}$, each of these females received a single intraperitoneal injection of ${ }^{3} \mathrm{HT}(5 \mu \mathrm{Ci} / \mathrm{gm}$ body weight).

The offspring included in this study were born after 69 or 70 days of gestation and housed with their mothers until they were given an overdose of sodium pentobarbital at $30 \mathrm{~d}$ of age and perfused intracardially with $10 \%$ neutral formalin. The brains were then removed and suspended in fresh formalin for 2 weeks, dehydrated in graded alcohols, and embedded in paraffin. The brains were sectioned frontally at $8 \mu \mathrm{m}$ and serial sections through the MPA were mounted on subbed slides. The slides were coated for autoradiography by dipping in Kodak NTR-2 emulsion diluted 1:1 with distilled water and exposed for 8 weeks in desiccated boxes at $4^{\circ} \mathrm{C}$. The slides were then developed with Kodak Dektol, stained with thionin, and coded so that the sex and injection date of the preparations were not known to the investigators.

Thirty-three brains were examined in this study: 2 male brains from each injection day except $E 28$, for which only 1 male brain was available, and 1 female brain from each day, except E29 and E30, for which no female brains were available. Cells in 8 cytoarchitectonic divisions were examined for silver grains: MPNp, MPNa, MPNc, Pea, suprachiasmatic nucleus (SCN), supraoptic nucleus (SON), and Bleier's (1983) preventricular periventricular nucleus (Pep). Pep was subdivided into median and lateral portions so that our results in the guinea pig could more easily be compared with the results of Altman and Bayer (1978a) in the rat. The median portion of Pep corresponds to the median preoptic nucleus (MnPO) of the Altman and Bayer study. The cell groups included in the present study are shown schematically in Figure 1.

Cells that fell within fields $\left(0.04 \mathrm{~mm}^{2}\right)$ cstablished by an ocular reticlc and distributed systematically throughout each division were examined with a Zeiss microscope fitted with a $10 \times$ ocular and a $100 \times$ oil- 


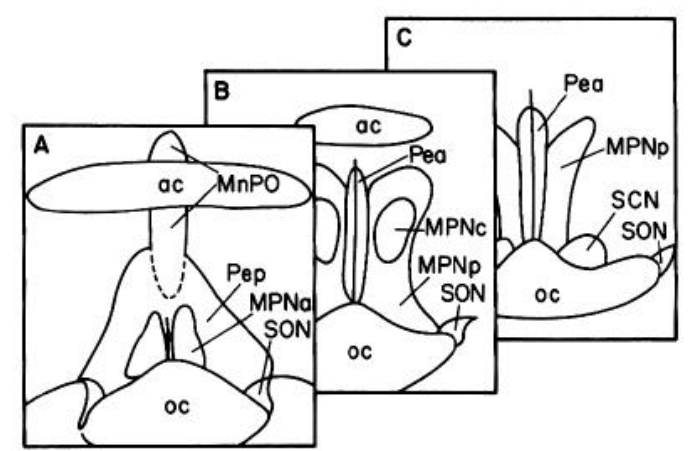

Figure 1. Schematic illustration of the nuclei examined in this study. Levels $a, b$, and $c$ correspond, respectively, to plates $6,9 \mathrm{a}$, and 11 in Bleier's (1983) cytoarchitectonic atlas of the guinea pig hypothalamus. Abbreviations: $a c$, anterior commissure; $M n P O$, median preoptic nucleus; $M P N a$, anterior compact subnucleus of the medial preoptic nucleus (MPN); $M P N c$, central compact subnucleus of the MPN; $M P N p$, principal portion of the MPN; oc, optic chiasm; $P e a$, anterior periventricular nucleus; $P e p$, preventricular periventricular nucleus; $S C N$, suprachiasmatic nucleus; $S O N$, supraoptic nucleus.

immersion objective. Based on a prior analysis of cell densities, the number of fields to be examined was chosen so that the number of cells sampled in each cytoarchitectonic division was approximately 100 per brain. Cells containing 5 or more silver grains were considered to be labeled above background, and cells containing 25 or more silver grains were considered to be heavily labeled. The criterion for background was derived by scanning the ventricular lumen. Very rarely within the lumen, 3 or 4 , but never 5 , silver grains were found clustered together within an area of $16 \mu \mathrm{m}^{2}$ (approximately the size of an average nucleus). The criterion for heavily labeled cells was established from a prior analysis of grain counts in 735 cells selected randomly from brains from each injection day. From this analysis, 100 was taken as the approximate maximum amount of label a cell could incorporate. After the incorporation by a cell of a maximal amount of label, each of its progeny would theoretically contain 25 silver grains following 2 mitotic divisions. There are many factors that act to reduce the amount of label incorporated by dividing neuroblasts (for review, see Sidman, 1970); nevertheless, the proportion of heavily labeled neurons within a given nucleus should provide an index of the proportion of cells in that nucleus that underwent their final division shortly after the ${ }^{3} \mathrm{HT}$ injection. The proportion of lightly labeled cells is an indicator of the proportion of cells that either underwent several divisions after the injection or incorporated a submaximal amount of ${ }^{3} \mathrm{HT}$ initially. Two heavily labeled neurons and the low incidence of background labeling are shown in Figure 2.

\section{Results}

Adequate numbers of male and female specimens were not available for a statistical evaluation of possible sex differences in the temporal or quantitative patterns of neurogenesis; however, examination of the available material suggests that such differences, if they exist, are not dramatic. Histograms showing the percentages of labeled and heavily labeled neurons from each day of gestation from E20 through E31 are shown in Figure 3. The injection made on E30 is presumed to have been faulty since no labeled neurons or glia were seen anywhere within the brains of animals exposed to ${ }^{3} \mathrm{HT}$ on this day and since label was seen in the brains of animals exposed on E31. Figure 3 shows that neurogenesis in the nonsexually dimorphic portions of the MPA is almost complete by E25, approximately the day that the fetal testis becomes active (Resko, 1970).

In contrast, $25-40 \%$ of the cells in the sexually dimorphic nuclei incorporate label on E25. The pattern of labeling seen in male and female guinea pigs following exposure to ${ }^{3} \mathrm{HT}$ on E26 is shown in the photomicrographs of Figures 4 and 5. Although

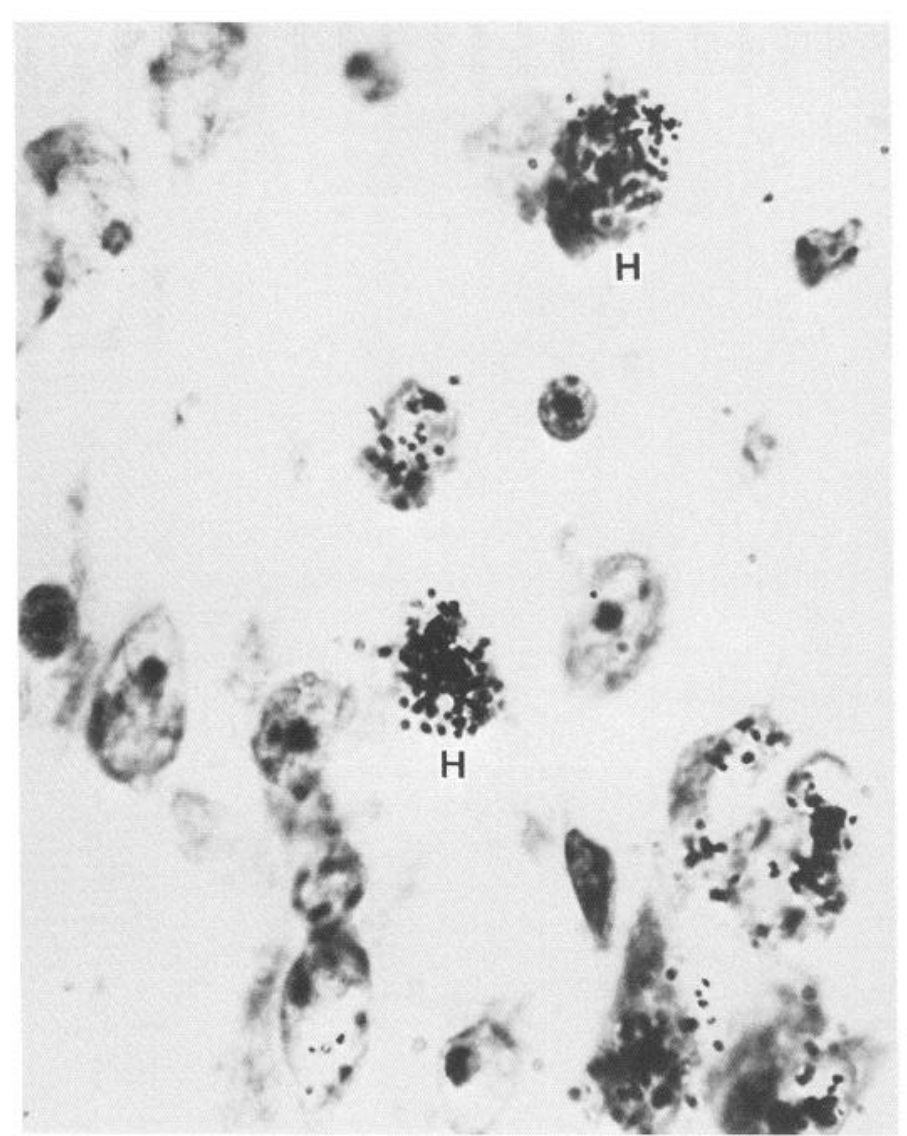

Figure 2. Photomicrograph $(\times 1500)$ of cells in the dorsal portion of MPNp of a female guinea pig exposed to ${ }^{3} \mathrm{HT}$ on E23. The figure shows 2 heavily labeled neurons $(H)$.

the female brain was sectioned more obliquely, the distribution of labeled cells was the same in both sexes. In both sexes exposed to ${ }^{3} \mathrm{HT}$ on this day, label is conspicuously more concentrated in the sexually dimorphic nuclei than in the surrounding MPA, and the labeled cells form a continuum extending posterodorsolaterally from MPNa to BNST. Although silver grains were not counted in the cells of the BNST, it is apparent by inspection of Figures $4 c$ and 5 that this nucleus becomes densely labeled by an injection on E26, suggesting that its pattern of neurogenesis is similar to that of the other cell groups in the region that display sexual dimorphism.

\section{Discussion}

The major finding of the present study is that the sexually dimorphic cell groups within the MPA are distinguished from their surround by their pattern of neurogenesis. Specifically, in contrast to cells destined for nonsexually dimorphic nuclei, a significant proportion of cells destined for the sexually dimorphic nuclei arises after the onset of fetal gonadal activity. The significance of this finding lies in the fact that gonadal steroids may influence the proliferation and differentiation of cells destined for the sexually dimorphic nuclei since receptors for gonadal steroids have been demonstrated in hypothalamic extracts from embryonic rodents (Vito and Fox, 1977, 1979; Vito et al., 1979 ) and since the sexually dimorphic nuclei (MPNa, MPNp, MPNc, Pea, BNST) have been shown to possess receptors for gonadal hormones in adulthood and during the period of sexual 
differentiation of the brain (Sar and Stumpf, 1975; Sheridan et al., 1975; Stumpf et al., 1975; Warembourg, 1977).

Our results suggest that the ncurogencsis of particular nuclei follows the same temporal sequence in the guinea pig as in the rat. In the rat, Altman and Bayer (1978a) reported the last day of neuronal proliferation for SON, MnPO, SCN, and Pea, respectively, to be gestational days $15,16,17$, and 17 . Our results in the guinea pig show the last day of proliferation for the same nuclei to be gestational days $26,26,27$, and 29 , respectively. According to Altman and Bayer, the last day on which cells became labeled in the MPA was E16, which corresponds approximately to E26 in the guinea pig. Jacobson and Gorski (1981), however, reported that neurogenesis in the SDN-POA (our MPNc) of the rat continues until at least E18, and that the MPNc is the only nucleus within the MPA labeled by the administration of ${ }^{3} \mathrm{HT}$ at this time. Assuming that the temporal patterns of neurogenesis are similar for rats and guinea pigs, our results are slightly at odds with both reports. Our study suggests that in the guinea pig proliferation of cells destined for MPNp and MPNc ceases approximately concurrently around E31 (which in the rat would correspond approximately to a time between 18-21 d postconception). We did not, however, actually determine the last day of neuronal production in either cell group since both incorporated label on our last day of injection. In a recent report by Jacobson et al. (1985), a dark-field photomicrograph of a section taken from a rat exposed to ${ }^{3} \mathrm{HT}$ on E18 (their fig. 2) shows a significant amount of label ventral to the MPNc (perhaps in the MPNp, depending on the precise tilt of the plane of sectioning), in addition to label within the MPNc. The authors do not comment on the discrepancy between this photomicrograph and their previous report. Perhaps this discrepancy is due to the difference in the ages of the subjects in their 2 studies (30 and $9 \mathrm{~d}$ after birth for the 1981 and 1985 studies, respectively). Alternatively, the discrepancy may be explained by the fact that in their earlier study the entire MPA was not examined. Because Jacobson et al. employ the de Groot plane, their sections, which pass through the MPNc dorsally, usually pass more ventrally through the anterior hypothalamic region overlying the SCN rather than through the MPNp. Thus, in their earlier study in which label in the MPNc was compared with label in an immediately more lateral region, label in the MPNa and MPNp may have been missed. The work of Jacobson et al. together with that of Altman and Bayer demonstrates that neurogenesis in the nonsexually dimorphic portions of the MPA ends at E16, the day that other workers have suggested that the fetal rat testis first becomes active (for references, see Baum, 1979). Thus, in the rat as well as the guinea pig there is a close temporal correspondence between the onset of fetal gonadal activity and the end of the proliferative period for neurons destined for nonsexually dimorphic regions of the MPA.

Hypothalamic sexual dimorphisms in rodents provide powerful model systems for the study of hormonal influences on neural development. At least 4 developmental processes may contribute to the establishment of sexual dimorphisms in hypothalamic cytoarchitecture. These are (1) neuronal proliferation, (2) neuronal growth, (3) neuronal migration, and (4) differential neuronal death. The possibility that each of these processes participates in sexually dimorphic development will be discussed in turn.

Sex differences in neuronal production have been described in the medial preoptic area of rats. More neurons were produced
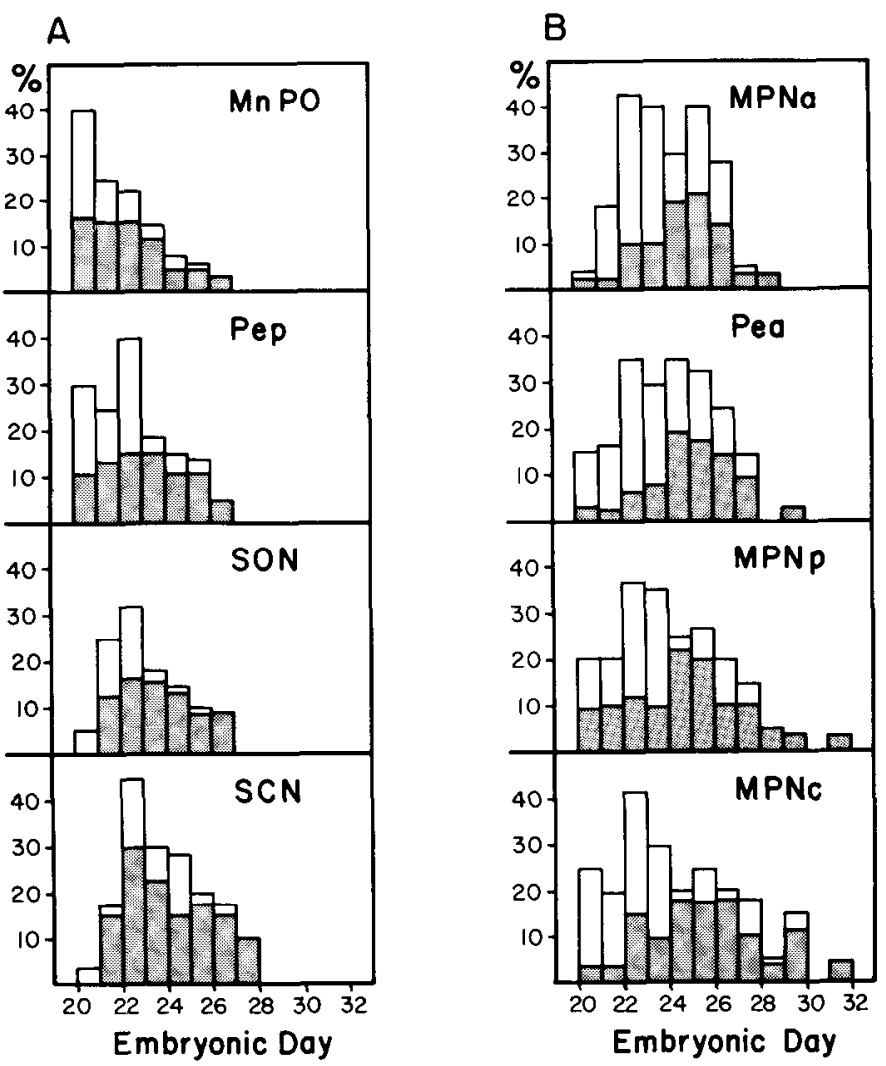

Figure 3. Percentage of cells labeled by ${ }^{3} \mathrm{HT}$ injection on the days indicated. The height of each bar represents the percentage of cells labeled above background, and the shaded portion represents the percentage of heavily labeled cells. Data from nonsexually dimorphic nuclei are included under $A$, while data from the sexually dimorphic nuclei are included under $B$. Abbreviations as in Figure 1 legend.

on E14 in females than in males, while the reverse was true for E17 (Jacobson and Gorski, 1981). In the present study no sex differences in neurogenesis were observed; however, only dramatic differences would have been detected given the present experimental protocol.

Sex differences in cell size are found within the sexually dimorphic regions of the guinea pig brain, but these appear to be related to the larger size of the male brain rather than to the dimorphisms per se. That is, cell size throughout the MPA tends to be larger in males than in females, even in the MPNa, which occupies a larger volume in females (Byne, 1985). Pfaff (1966) also concluded that the larger neuronal size of male rats relative to females is related to the larger brain size of males.

Cells that form the MPA proliferate in the germinal layer of the third ventricular lining and subsequently migrate to their final positions (Altman and Bayer, 1978b). In the rat, presumptive MPNc cells migrate dorsally from the ventral portion of the ventricular lining during the early postnatal period (Jacobson et al., 1985). This finding, together with the fact that cells in the MPA of guinea pigs appear to be concentrated more anteroventrally in females than in males (Byne and Bleier, 1987, Fig. 2) suggests the possibility that testosterone stimulates the posterodorsal migration of a certain proportion of presumptive MPNc cells. In other words, in the absence of a timely and potent androgenic stimulus, cells would retain a more anteroventral localization. Jacobson et al. (1985) failed to detect a sex difference in neuronal migration in the rat; however, such a 

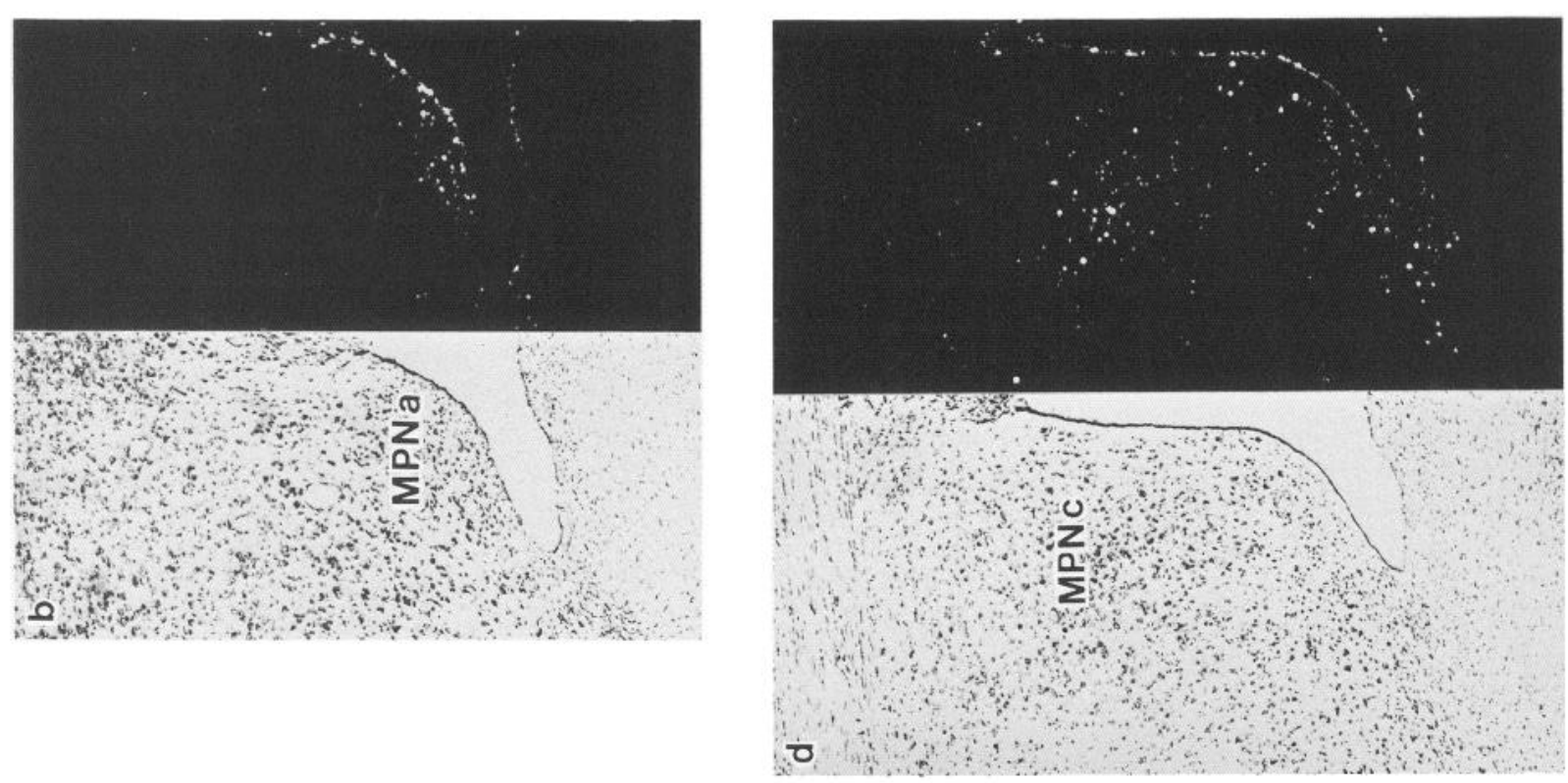

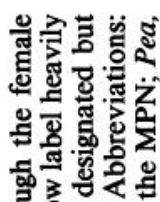

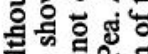

त्ञ

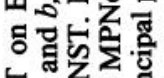

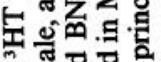

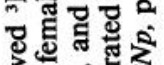

\& of

政

의

के

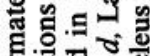

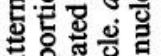

크원은

흥

른

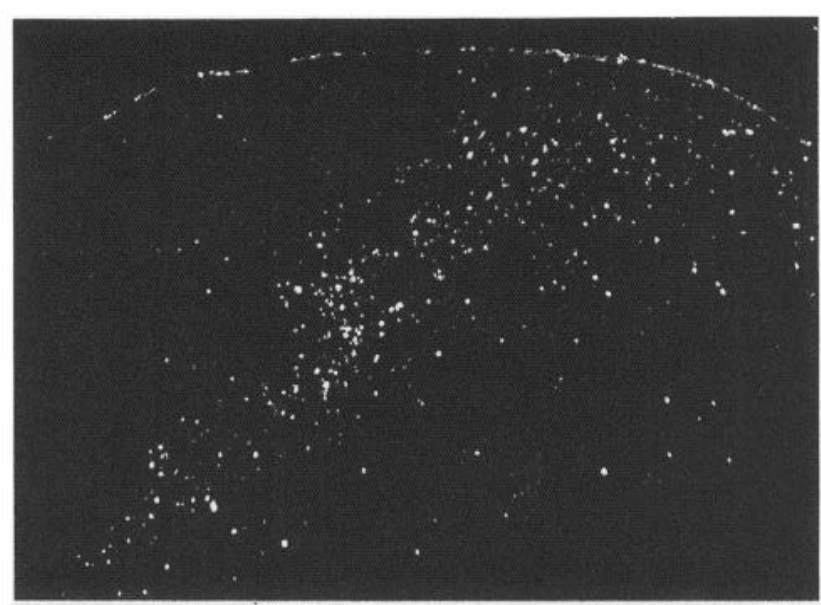

명

อ

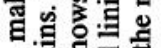

명혀

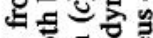

등. 댈 융

들

.

.

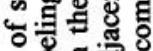

ठ응

×

E
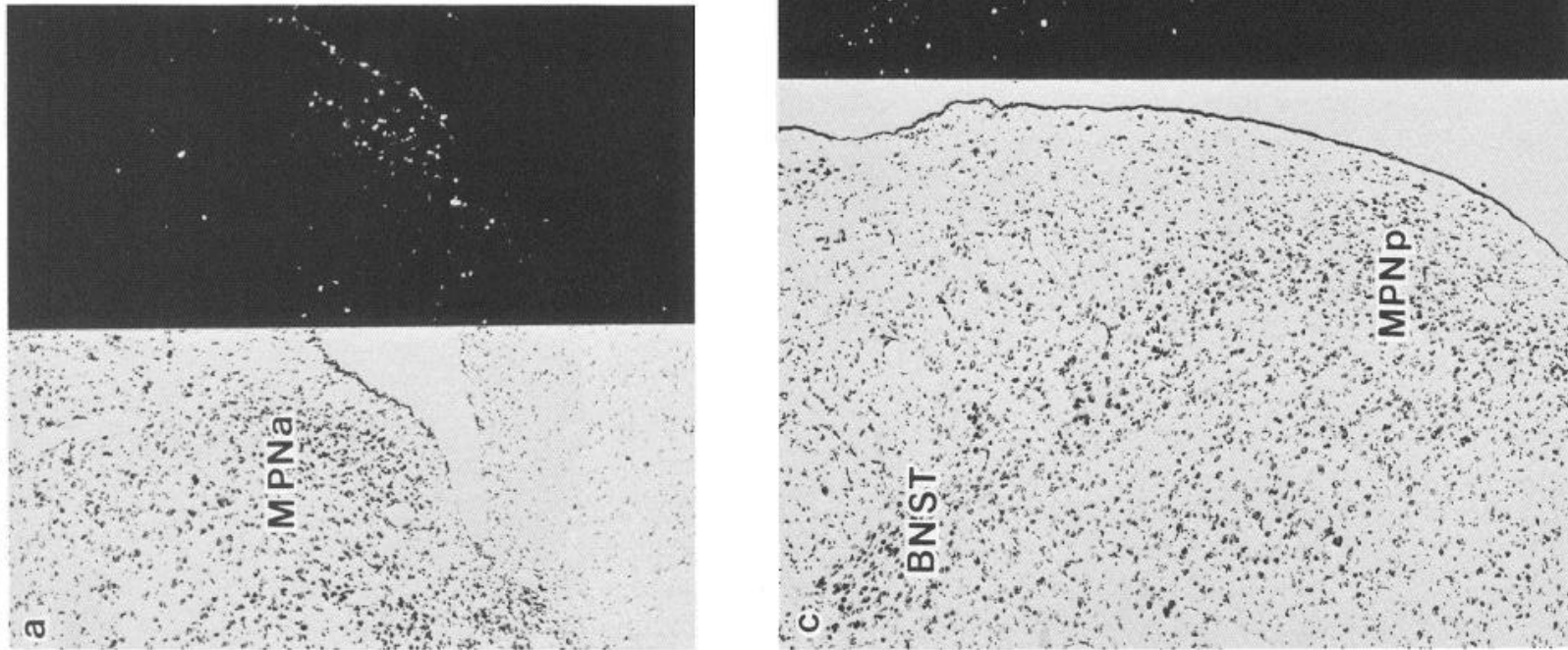

解

항 을

瓷席

정

춤정

을

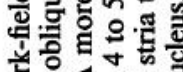

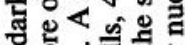

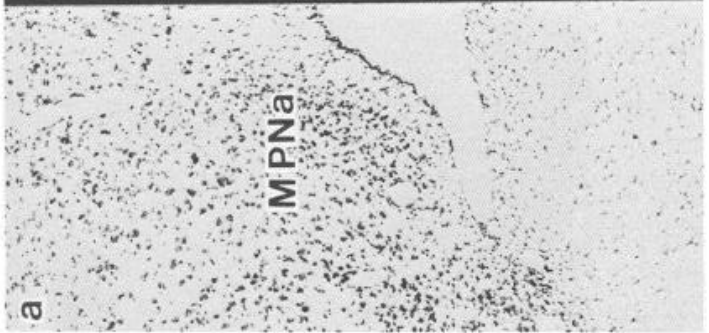

7 융

政

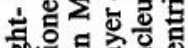

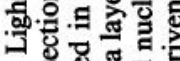

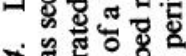

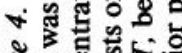

5. 8.5

ไี้ 

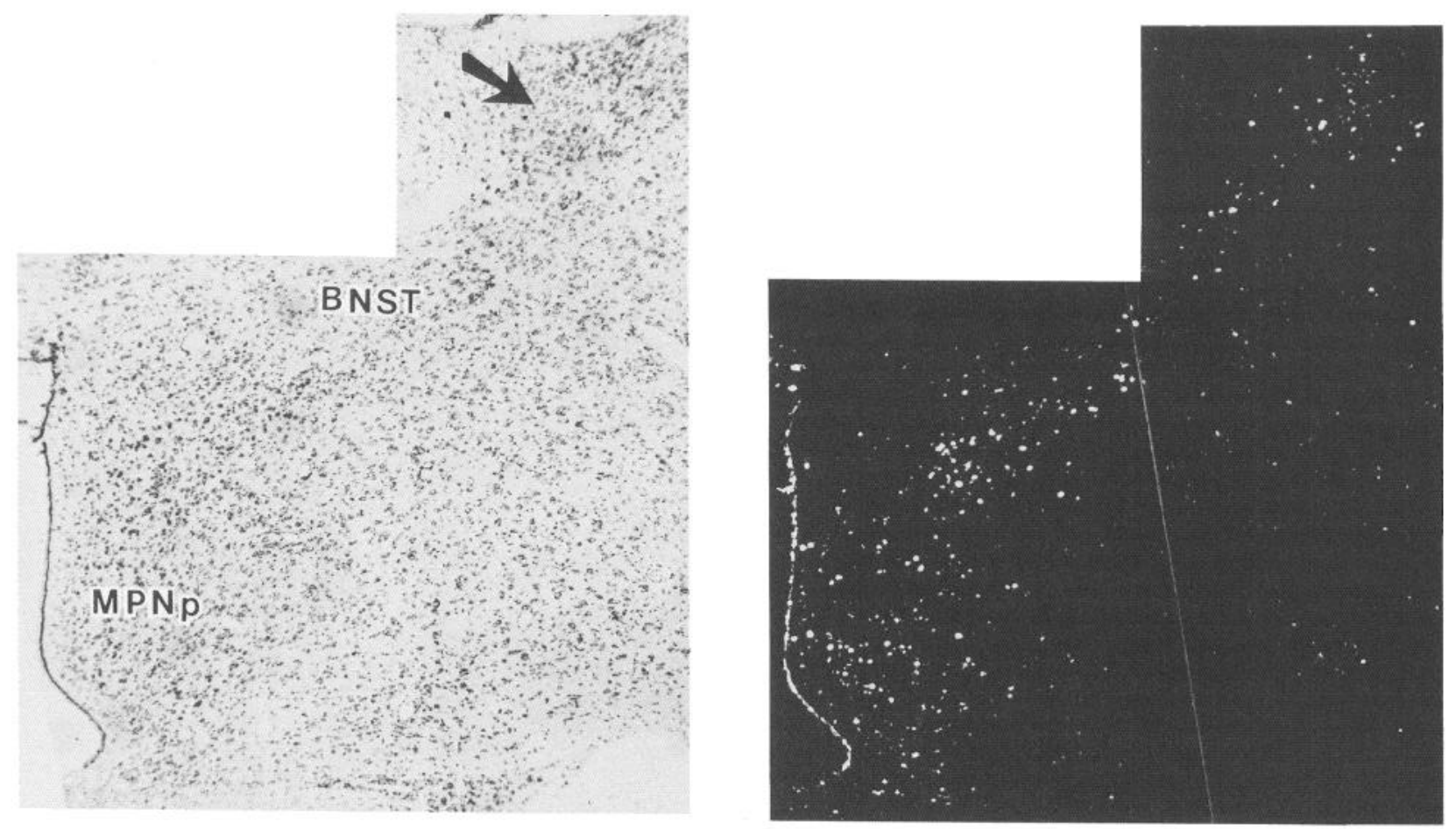

Figure 5. Photomicrographs $(\times 60)$ of a more caudal section through the hypothalamus of the male in Figure 3 . Label is concentrated in Pea, MPNp, and BNST. The densely staining portion of the BNST (arrow) appears to correspond to the portion shown to be sexually dimorphic by Hines et al. (1985). Abbreviations as in Figure 4 legend.

difference would be extraordinarily difficult to demonstrate due to the fact that the contours of the brain itself are changing during the migratory period.

Sex differences in selective cell death have been shown to participate in establishing cytoarchitectonic sex differences in the gerbil brain (Ulibarri and Yahr, 1984) and in the rat spinal cord (Nordeen et al., 1984). In these cases, testosterone was shown to decrease the incidence of selective cell death. Testosterone also inhibits the neuronal loss that normally occurs in the superior cervical ganglion during development (Wright et al., 1983). While such an action of testosterone could easily explain why particular nuclei are larger in males than in females, it cannot easily explain why the MPNa is larger in females. Estrogen and testosterone exert similar organizational actions on the developing rat brain of some species (see Goy and McEwen, 1980). It is unlikely, however, that sex differences in estrogen production by the fetal gonads contribute to the sexually dimorphic development of the MPNa or other sexually dimorphic nuclei in the guinea pig since estrogen levels are extremely low or below the level of assay sensitivity throughout the period of sexual differentiation (Buhl et al., 1979). Sex differences in progesterone levels were also not found during this period.

In summary, the proliferation of cells destined for sexually dimorphic hypothalamic nuclei is still in progress after the commencement of fetal gonadal activity and during the early portion of the period in which androgen exposure has been shown to masculinize hypothalamic cytoarchitecture of female guinea pigs (Byne and Bleier, 1987). Thus, the neurogenesis and subsequent developmental processes of sexually dimorphic nuclei may be influenced by gonadal steroids. Now that the period of medial preoptic neurogenesis has been established, further work may elucidate the mechanisms involved in the development of sex- ually dimorphic nuclei in the guinea pig. Although much of our knowledge of neural sexual differentiation comes from studies of altricial rodents, the guinea pig may provide a preferable model for particular aspects of primate reproductive development (see Terasawa et al., 1984; Hines et al., 1985; Byne and Bleier, 1987). Extrapolating from work done on the rat, we suggest that sex differences in neuronal production and migratory patterns may contribute to establishing a larger MPNa in females, while these developmental processes together with sex differences in selective cell death may contribute to establishing a larger MPNc in males.

\section{References}

Altman, J., and S. A. Bayer (1978a) Development of the diencephalon of the rat: I. Autoradiographic study of the time of origin and settling patterns of neurons of the hypothalamus. J. Comp. Neurol. 182: $945-$ 972.

Altman, J., and S. A. Bayer (1978b) Development of the diencephalon of the rat: II. Correlation of the embryonic development of the hypothalamus with the time of origin of its neurons. J. Comp. Neurol. 182: 973-993.

Baum, M. J. (1979) Differentiation of coital behavior in mammals: A comparative analysis. Neurosci. Biobeh. Rev. 3: 265-284.

Bleier, R. (1983) The Hypothalamus of the Guinea Pig: A Cytoarchitectonic Atlas, University of Wisconsin Press, Madison, WI.

Bleier, R., W. Byne, and I. Siggelkow (1982) Cytoarchitectonic sexual dimorphisms of the medial preoptic and anterior hypothalamic areas in the guinea pig, rat, hamster and mouse. J. Comp. Neurol. 212: $118-130$.

Buhl, A. E., L. M. Pasztor, and J. A. Resko (1979) Sex steroids in guinea pig fetuses after sexual differentiation of the gonads. Biol. Reprod. 21: 905-909.

Byne, W. (1985) Cytoarchitectonic sexual dimorphisms in the guinea pig: Morphometric, functional and ontogenetic studies. Ph.D. thesis, University of Wisconsin, Madison, WI.

Byne, W., and Bleier, R. (1987) Medial preoptic sexual dimorphisms 
in the guinea pig: I. An investigation of their hormonal dependence. J. Neurosci. 7: 2688-2696.

Gorski, R. A., J. H. Cordon, J. E. Shryne, and A. M. Southam (1978) Evidence for a morphological sex difference within the medial preoptic area of the rat brain. Brain Res. 148: 333-346.

Goy, R. W., and B. S. McEwen (1980) Sexual Differentiation of the Brain, MIT Press, Cambridge, MA.

Hines, M. F. C. Davis, A. Coquelin, R. W. Goy, and R. A. Gorski (1985) Sexually dimorphic regions in the medial preoptic area and the bed nucleus of the stria terminalis of the guinea pig brain. J. Neurosci. 5: 40-47.

Ifft, J. D. (1972) An autoradiographic study of the time of final divisions of neurons in rat hypothalamic nuclei. J. Comp. Neurol. 144: 193-204.

Jacobson, C. D., and R. A. Gorski (1981) Neurogenesis of the sexually dimorphic nucleus of the preoptic area of the rat. J. Comp. Neurol. 196: 519-529.

Jacobson, C. D., F. C. Davis, and R. A. Gorski (1985) Formation of the sexually dimorphic nucleus of the preoptic area: Neuronal growth, migration and changes in cell number. Dev. Brain Res. 21: 7-18.

Nordeen, E. J., K. W. Nordeen, D. R. Singelaub, and A. P. Arnold (1984) Ontogeny of sexual dimorphism in a rat spinal nucleus: $I$. Hormonal control of neuronal number. Soc. Neurosci. Abstr. 7: 722.

Pfaff, D. W. (1966) Morphological changes in the brains of adult male rats after neonatal castration. J. Endocrinol. 36: 415-416.

Resko, J. A. (1970) Androgens in systemic plasma of male guinea pigs during development and after castration in adulthood. Endocrinology 86: 1444-1447.

Sar, M., and W. E. Stumpf (1975) Cellular localization of progestin estrogen in guinea pig hypothalamus by autoradiography. In Anatomical Neuroendocrinology, W. E. Stumpf and L. D. Grant, eds., pp. 142-152, Karger, New York.
Sheridan, P. J., M. Sar, and W. E. Stumpf (1975) Estrogen and androgen distribution in the brain of neonatal rats. In Anatomical Neuroendocrinology, W. E. Stumpf and L. D. Grant, eds., pp. 134-141, Karger, New York.

Sidman, R. L. (1970) Autoradiographic methods and principles for study of the nervous system with tritiated thymidinc. In Contemporary Research Methods in Neuroanatomy, W. J. H. Nauta and S. O. E. Ebbesson, eds., pp. 252-274, Springer-Verlag, New York.

Stumpf, W. E., M. Sar, and D. A. Keffer (1975) Atlas of estrogen target cells in the rat brain. In Anatomical Neuroendocrinology, W. E. Stumpf and L. D. Grant, eds., pp. 104-1 19, Karger, New York.

Terasawa, E., R. R. Yeoman, and N. J. Shultz (1984) Factors influencing the progesterone-induced luteinizing hormone surge in rhesus monkeys: Diurnal influence and time interval after estrogen. Biol. Reprod. 31: 732-741.

Ulibarri, C., and P. Yahr (1984) Sexual differentiation of the gerbil brain may involve selective cell death in females. Soc. Neurosci. Abstr. 10: 454.

Vito, C. C., and T. O. Fox (1977) Embryonic estradiol receptors from mouse and rat brain. Soc. Ncurosci. Abstr. 3: 359.

Vito, C. C., and T. O. Fox (1979) Embryonic rodent brain contains estrogen receptors. Science 204: 517-519.

Vito, C. C., S. J. Wieland, and T. O. Fox (1979) Androgen receptors exist throughout the "critical period" of brain sexual differentiation. Nature 282: 308-310.

Warembourg, M. (1977) Radioautographic localization of estrogenconcentrating cells in the brain and pituitary of the guinea pig. Brain Res. 123: 357-362.

Wright, L. L., P. Beaston-Wimmer, and A. J. Smoley (1983) Developmental plasticity of sympathetic neurons induced by gonadal steroids. Soc. Neurosci. Abstr. 9: 306. 